\title{
Article \\ Cooperative Spectrum Sensing Based on Convolutional Neural Networks
}

\author{
Youheng Tan and Xiaojun Jing *
}

\section{check for}

updates

Citation: Tan, Y.; Jing, X.

Cooperative Spectrum Sensing Based on Convolutional Neural Networks.

Appl. Sci. 2021, 11, 4440.

https://doi.org/10.3390/app11104440

Academic Editor: Youngchul Bae

Received: 16 March 2021

Accepted: 28 April 2021

Published: 13 May 2021

Publisher's Note: MDPI stays neutral with regard to jurisdictional claims in published maps and institutional affiliations.

Copyright: (c) 2021 by the authors. Licensee MDPI, Basel, Switzerland. This article is an open access article distributed under the terms and conditions of the Creative Commons Attribution (CC BY) license (https:// creativecommons.org/licenses/by/ $4.0 /)$.
School of Information and Communication Engineering, Beijing University of Posts and Telecommunications, Beijing 100876, China; yh_tan@bupt.edu.cn

* Correspondence: jxiaojun@bupt.edu.cn

\begin{abstract}
Cooperative spectrum sensing (CSS) is an important topic due to its capacity to solve the issue of the hidden terminal. However, the sensing performance of CSS is still poor, especially in low signal-to-noise ratio (SNR) situations. In this paper, convolutional neural networks (CNN) are considered to extract the features of the observed signal and, as a consequence, improve the sensing performance. More specifically, a novel two-dimensional dataset of the received signal is established and three classical CNN (LeNet, AlexNet and VGG-16)-based CSS schemes are trained and analyzed on the proposed dataset. In addition, sensing performance comparisons are made between the proposed CNN-based CSS schemes and the AND, OR, majority voting-based CSS schemes. The simulation results state that the sensing accuracy of the proposed schemes is greatly improved and the network depth helps with this.
\end{abstract}

Keywords: cooperative spectrum sensing; convolutional neural networks; LeNet; AlexNet; VGG-16

\section{Introduction}

The rapid development of wireless communication technology has led to more and more wireless network services. The radio spectrum, as the most valuable resource in wireless networks, cannot meet the requirements of wireless services at present and in the future [1]. The existing fixed spectrum allocation method makes the spectrum utilization low and seriously uneven. According to the investigation, the average spectrum utilization is less than $5 \%$ at any time or place [2]. Dynamic spectrum access (DSA) is considered to be the main technical solution to the contradiction between supply and demand [3]. As the basis of DSA, cognitive radio (CR) technology has become one of the most cutting-edge research topics in the field of wireless networking.

$\mathrm{CR}$ has the ability to interact with its communication environment and change its transmission parameters according to the result. The cognitive radio network (CRN) [4-6] is a network with $\mathrm{CR}$ as the service terminal. In the $\mathrm{CRN}$, the licensed user and the unlicensed user coexist, where the licensed user is named the primary user (PU) and the unlicensed user is called the secondary user (SU). The CR users with CR can sense idle spectrum resources and maximize the utilization of spectrum resources without interfering with PU. The research work of the CRN is mainly focused on spectrum sensing (SS), spectrum sharing, spectrum access and spectrum mobility [7]. Different from the channel allocation of traditional wireless networks, the channel allocation of the CRN is based on the real-time sensing of channel conditions [8]. As a result, $\mathrm{SS}$ is the foundation of CR.

The main function of SS is to detect the spectrum holes available to SU and to monitor the signal activity of the PU to ensure that when the PU uses the spectrum again [9], SU can quickly exit the corresponding frequency band. Two factors influence the performance of SS, namely false alarm probability and detection probability. The false alarm probability is negatively correlated with the throughput of the $C R$, while the detection probability denotes the protection capacity for PU. Classical SS schemes contain single node-based 
SS and cooperative spectrum sensing (CSS). Single node-based SS contains matched filter detection [10], energy detection (ED) [11], cyclostationary feature detection [12] and covariance matrix detection [13], etc. However, the single node-based SS either has poor performance or has high complexity [7]. In the case of the hidden terminal issue (the shadow and the deep fading), the sensing results of the single node-based SS are not reliable, so it is necessary to integrate the sensing results of multiple nodes in order to improve the reliability of detection, namely CSS.

Classical CSS schemes contain centralized CSS and distributed CSS [14-18]. Centralized CSS denotes that each node aggregates the local sensing information to the fusion center, and the fusion center makes a decision according to the fusion rules. Finally, the fusion center (FC) feeds back the decision result to each local node $[15,16]$. According to the fusion rule at the FC, centralized CSS contains the AND rule-based CSS, the OR rule-based CSS and the majority voting (MV)-based CSS, etc. There is no fusion center for distributed CSS $[17,18]$, and each local node exchanges the detection results with the others, and then combines the local fusion decision. The sensing accuracy of the CR system is effectively improved for the distributed CSS [19-21]. However, this is at the expense of network burden and system overhead [22-24]. In consequence, centralized CSS is mainly considered in this paper.

With the rapid development of artificial intelligence (AI) technology, deep learning has attracted much attention due to its capacity of automatic feature extraction [25-27]. As one of the most successful technologies of AI, the convolution neural network (CNN) has been successfully applied to the field of wireless communication [28,29]. As a result, this paper is devoted to the CNN-based CSS for the possible performance improvement. This paper considers the scenario where the received energy vector of each node is sent to the FC and a two-dimensional matrix is formed at the FC. Then, the covariance matrix of this two-dimensional matrix is obtained and the mean value of the covariance matrix is removed. After this, the updated covariance matrix is input to the $\mathrm{CNN}$ and the decision result is obtained. Finally, the FC feeds back the decision result to each local node. The main work and contributions of this paper are as follows:

1. A novel two-dimensional dataset of the received signal is established for CSS and the signal-to-noise ratio (SNR) of the dataset varies from $-8 \mathrm{~dB}$ to $5 \mathrm{~dB}$ with an SNR step of $1 \mathrm{~dB}$.

2. The classical LeNet network, AlexNet network and VGG-16 network are trained on the proposed dataset. The corresponding false alarm probability and detection probability of the three networks are analyzed and compared.

3. The AND rule-based CSS, the OR rule-based SS and the MV-based CSS are compared with the proposed CNN-based CSS scheme. The experimental results validate the effectiveness of the proposed scheme.

The rest of this paper is organized as follows: Section 2 gives the related work of this paper. In Section 3, the sensing scenario and sensing model are discussed. The main contribution of this paper is shown in Section 4. Simulation experiments and result analysis are presented in Section 5. Finally, Section 6 concludes this paper.

\section{Related Work}

In this section, the machine learning-based SS schemes are reviewed and discussed. 
Table 1. The machine learning-based SS schemes.

\begin{tabular}{|c|c|c|}
\hline Reference & Main Contribution & Advantages \\
\hline$[30]$ & $\begin{array}{l}\text { A STFT-CNN method is proposed for SS based on the short-term } \\
\text { Fourier transform (STFT) and CNN. }\end{array}$ & $\begin{array}{l}\text { The signal feature in the frequency } \\
\text { domain is considered. }\end{array}$ \\
\hline$[31]$ & $\begin{array}{l}\text { The application of machine learning (ML) models in cooperative } \\
\text { spectrum sensing of cognitive radio networks (CRNs) is considered, } \\
\text { including multilayer perceptron (MLP), support vector machine and } \\
\text { Naive Bayes. }\end{array}$ & $\begin{array}{l}\text { The sensing complexity is much lower } \\
\text { compared with the ML-based schemes. }\end{array}$ \\
\hline$[32]$ & $\begin{array}{l}\text { A CNN-LSTM detector is proposed, which first uses the CNN to } \\
\text { extract the energy correlation features from the covariance matrices } \\
\text { generated by the sensing data; then, the series of energy correlation } \\
\text { features corresponding to multiple sensing periods are input into the } \\
\text { LSTM so that the PU activity pattern can be learned. }\end{array}$ & $\begin{array}{l}\text { The time series features are considered } \\
\text { for the possible improvement of the } \\
\text { sensing performance. }\end{array}$ \\
\hline [33] & $\begin{array}{l}\text { The distributed deep reinforcement learning method is adopted to } \\
\text { learn the optimal CSS strategy. }\end{array}$ & $\begin{array}{l}\text { The distributed CSS is discussed and } \\
\text { analyzed. }\end{array}$ \\
\hline [34] & $\begin{array}{l}\text { Multiple machine learning-enabled solutions are adopted to tackle the } \\
\text { challenges of the complex sensing model in CSS for a non-orthogonal } \\
\text { multiple access transmission mechanism, including unsupervised } \\
\text { learning algorithms (K-means clustering and Gaussian mixture model) } \\
\text { as well as supervised learning algorithms (directed acyclic } \\
\text { graph-support vector machine, K-nearest-neighbor and } \\
\text { back-propagation neural network). }\end{array}$ & $\begin{array}{l}\text { The sensing accuracy is greatly improved } \\
\text { with a moderate complexity. }\end{array}$ \\
\hline [35] & $\begin{array}{l}\text { Deep reinforcement learning (DRL)-based CSS algorithm is proposed, } \\
\text { which is employed to decrease the signaling in the network of SUs. }\end{array}$ & $\begin{array}{l}\text { The sensing accuracy of CSS is improved } \\
\text { as far as possible. }\end{array}$ \\
\hline [36] & $\begin{array}{l}\text { Based on the fact that pulse radar signals can be modeled as a train of } \\
\text { rectangular pulses with two amplitude levels, we develop a generalized } \\
\text { likelihood ratio test spectrum sensing scheme, as well as its less complex } \\
\text { sub-optimal variations, to detect the presence of such signals. }\end{array}$ & $\begin{array}{l}\text { The closed-from solutions can be } \\
\text { obtained with a lower complexity. }\end{array}$ \\
\hline [37] & $\begin{array}{l}\text { A deep compressive spectrum sensing GAN (DCSS-GAN) is proposed, } \\
\text { where two neural networks are trained to compete with each other to } \\
\text { recover the spectrum from undersampled samples in the time domain. }\end{array}$ & $\begin{array}{l}\text { The sensing performance with the } \\
\text { undersampled samples can be greatly } \\
\text { improved. }\end{array}$ \\
\hline
\end{tabular}

From Table 1, it can be seen that much effort has been made to possibly improve the performance of SS. In [31,34,36], machine learning (ML)-based schemes are considered for SS, including multilayer perceptron (MLP), support vector machine, Naive Bayes, directed acyclic graph-support vector machine, K-nearest-neighbor and generalized likelihood ratio test. Meanwhile, deep learning (DL) is utilized for SS in $[30,32,33,35,37]$, where CNN with STFT, CNN with LSTM, deep reinforcement learning and a generative adversarial network (GAN) are considered.

Although the sensing performance is greatly improved for the above-mentioned schemes, the ML-based schemes are devoted to the extraction of the shallow features, while the DL-based schemes aim at feature extraction in the frequency domain and complex features, at the expense of computational complexity. In this paper, three basic CNN networks are considered to improve the performance of SS, based on a novel SS dataset. The LeNet network, AlexNet network and VGG-16 network are trained and adjusted on the proposed dataset. Superb sensing performance is obtained based on the simulation experiments.

Note that the proposed schemes in this paper balance sensing performance and sensing complexity. Although the considered CNN networks are classical, they are easy to implement due to their high popularity. In addition, the dataset considered in this paper is based on the energy value of the local SS node. Both the data acquisition and the network structure have low complexity. However, the sensing performance is at a high level according to the results of the simulation experiments. In summary, the proposed schemes in this paper are necessary and useful for the possible performance improvement of SS. 


\section{System Model}

\subsection{Sensing Scenario}

As shown in Figure 1, each SU directly transmits the local sensing information to the FC, and the FC makes the final decision and then sends it to each SU for the centralized CSS. The details of centralized CSS are as follows:

1. The energy vector of each sensing node is obtained by the sampling and signal processing.

2. The obtained energy vector of each node is sent to FC over the reporting channel, where the reporting channel obeys the Rayleigh distribution.

3. After the reporting channel, a two-dimensional matrix is obtained with the energy vector of each sensing node. Then, the mean value of the covariance of the twodimensional matrix is removed by each matrix element. Finally, the updated twodimensional matrix is input to the CNN module for the final decision.

4. The final decision result at FC is sent to each local sensing node.

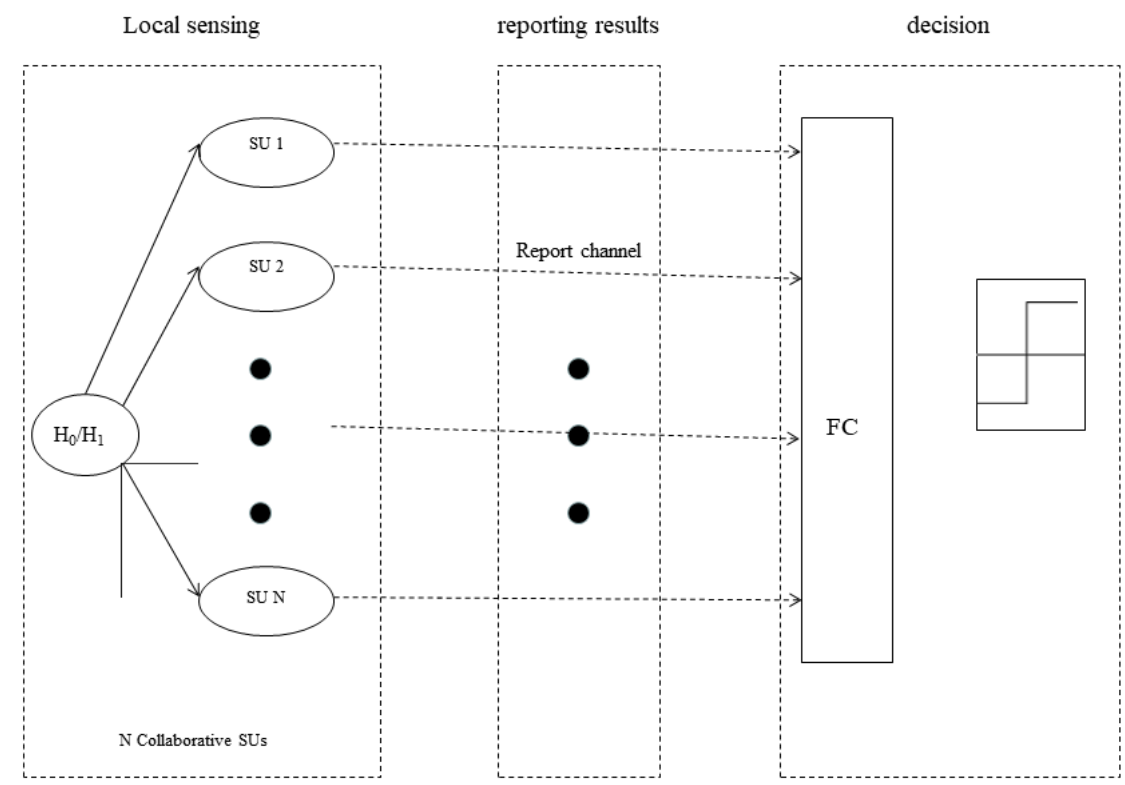

Figure 1. The flowchart of centralized CSS (cooperative spectrum sensing).

\subsection{Sensing Model}

Assume that the received signal at the $i$ th receiver of local sensing node can be formulated as:

$$
r(n)=h(n) s(n)+x(n)
$$

where $s(n)$ denotes the primary signal, $x(n)$ denotes the background noise with Gaussian distribution and $h(n)$ represents the Rayleigh channel. The sampling frequency is $f_{s}$ and the sensing duration is $\tau$.

The energy value of the $i$ th local sensing node can be written as shown in (2) and (3):

$$
\begin{gathered}
E_{r}^{i}(n)=\sqrt{\left|r_{1}(n)\right|^{2}+\left|r_{2}(n)\right|^{2}}, \\
r(n)=r_{1}(n)+j r_{2}(n),
\end{gathered}
$$

where $r_{1}(n)$ denotes the real part of $r(n)$ while $r_{2}(n)$ denotes its imaginary part.

Then, the energy vector can be obtained:

$$
\begin{gathered}
E_{r}^{i}=\left[E_{r}^{i}(1), \cdots, E_{r}^{i}(N)\right], \\
N=f_{s} \tau,
\end{gathered}
$$


where $N$ denotes the sampling point of the $i$ th local sensing node.

After the reporting channel, the energy vector can be updated as:

$$
\begin{gathered}
E_{r 1}^{i}=\left[E_{r 1}^{i}(1), \cdots, E_{r 1}^{i}(N)\right], \\
E_{r 1}^{i}(n)=h_{1}(n) E_{r}^{i}(n)+x_{1}(n), n=1, \cdots, N,
\end{gathered}
$$

where $h_{1}(n)$ denotes the reporting channel with the Rayleigh distribution and $x_{1}(n)$ denotes the background noise.

At the FC, the two-dimensional matrix is obtained based on each local energy vector:

$$
M=\left[\begin{array}{ccc}
E_{r 1}^{1}(1) & \cdots & E_{r 1}^{1}(N) \\
\vdots & \ddots & \vdots \\
E_{r 1}^{k}(1) & \ldots & E_{r 1}^{k}(N)
\end{array}\right]
$$

Based on (8), the covariance matrix of $M$ can be denoted as:

$$
R_{M}=\left[\begin{array}{ccc}
R_{M}(1,1) & \ldots & R_{M}(N, 1) \\
\vdots & \ddots & \vdots \\
R_{M}(1, N) & \ldots & R_{M}(N, N)
\end{array}\right]
$$

The updated covariance matrix with the mean value removed is shown as:

$$
R_{M 1}=\left[\begin{array}{ccc}
R_{M 1}(1,1) & \cdots & R_{M 1}(N, 1) \\
\vdots & \ddots & \vdots \\
R_{M 1}(1, N) & \ldots & R_{M 1}(N, N)
\end{array}\right]
$$

Finally, $R_{M 1}$ is input to the $\mathrm{CNN}$ module for the final decision and the decision result can be described as:

$$
D=\psi\left(R_{M 1}\right)
$$

where $\psi(\cdot)$ denotes the $\mathrm{CNN}$ operations.

\section{Main Contribution}

In this section, the main contribution of this paper is discussed, including the dataset construction, the $\mathrm{CNN}$ module considered in this paper and the training of the CNNs.

\subsection{Dataset Construction}

The simulation band is conducted at very high frequency (VHF), where the carrier frequency is set as $f_{c}=1.0 \times 10^{8} \mathrm{~Hz}$ and sampling frequency is $f_{s}=3.0 \times 10^{8} \mathrm{~Hz}$. Orthogonal Frequency Division Multiplexing (OFDM) signal is chosen as the test signal (PU) for its generality and popularity in wireless communication networks. In the simulation, the OFDM signal is first generated, and then white Gaussian noise with with the mean zero and the variance one is added into the OFDM signal.

In this paper, the sensing nodes are fixed to 5 without the description because the sensing node number determines the dataset construction. If the number of sensing nodes is not fixed, the required dataset is very large. On the other hand, the sensing node number determines the size of the two-dimensional matrix, and the sensing performance with the same CNN network will be relatively positive with the sensing node number. As a result, fixing the sensing nodes does not influence the performance validation of the proposed network.

For each sensing node, a random integer between -8 and 5 is used as the signal-tonoise ratio (SNR). There are two kinds of samples in the constructed dataset, namely the $H_{0}$ sample and the $H_{1}$ sample, where the $H_{0}$ sample denotes the absence of PU and the $H_{1}$ sample denotes the presence of PU. The $H_{0}$ sample and the $H_{1}$ sample are in equal proportion. For each sample (the $H_{0}$ sample or the $H_{1}$ sample), 6000 sets of the complex 
signal are obtained, with the sampling points of with each sampling points 1000 and then the modulus value is calculated for each sampling point. As a result, a modulus sequence matrix is obtained with the size $5 \times 1000$. According to the operations in (9) and (10), the updated covariance matrix is obtained. Then, the dataset construction is finished, where the first 2000 groups are taken as the test set and the rest work as the training set.

Figure 2 exhibits the obtained covariance matrix when $\mathrm{SNR}=-8 \mathrm{~dB}$, where the left figure denotes the $H_{0}$ case and the right one denotes the presence of PU. In Figure 2, much difference exists, which helps to determine the presence or absence of the PU.
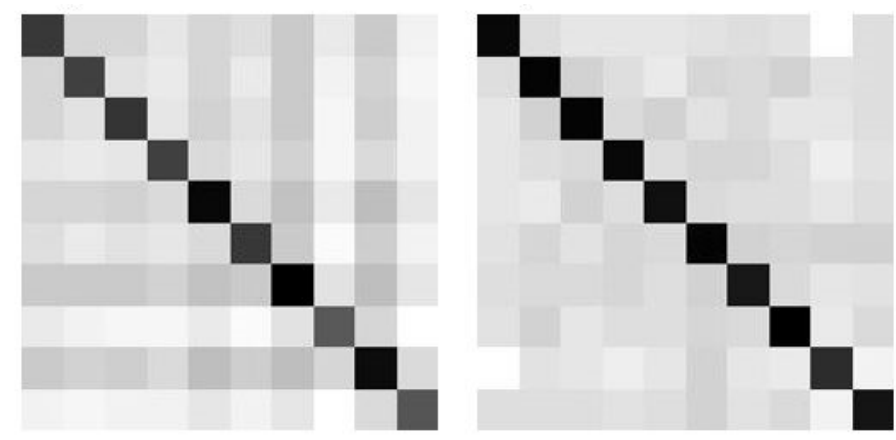

Figure 2. The dataset sample when $\mathrm{SNR}=-8 \mathrm{~dB}$.

\subsection{CNN Module}

In this subsection, three classical CNN models are considered for the CSS, namely LeNet, AlexNet and VGG-16 [38-40].

LeNet was proposed by LeCun, the founder of the convolution neural network, in 1994 to solve the visual task of handwritten digit recognition [38]. As shown in Figure 3, LeNet contains 2 convolution layers, 2 pooling layers and 2 full connection layers.

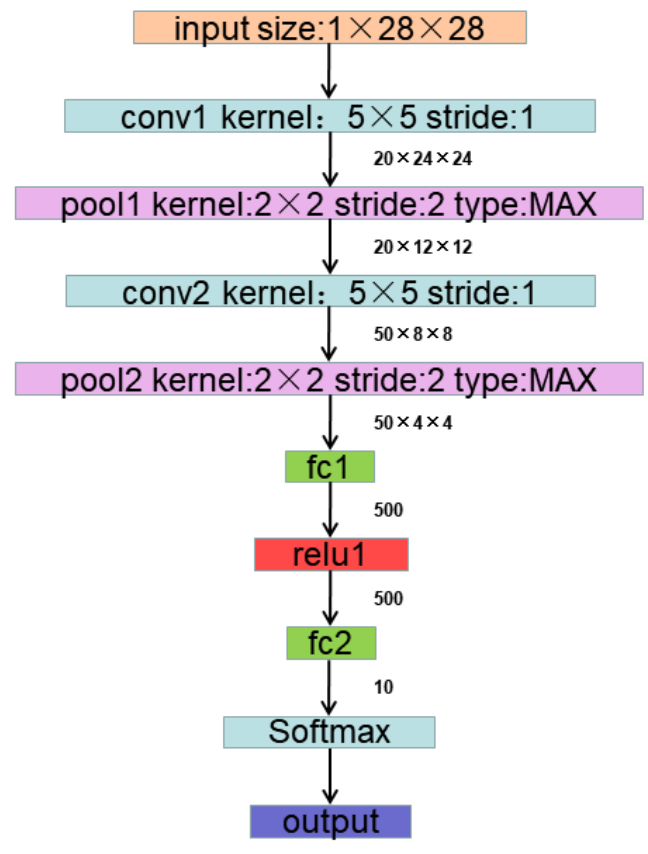

Figure 3. The network architecture of LeNet [38].

AlexNet carries forward the idea of LeNet and applies the basic principles of CNN to a very deep and wide network [39], as shown in Figure 4. The advantages of AlexNet can be described as five aspects. (1) ReLU is successfully used as the activation function of $\mathrm{CNN}$, and its effect is proven to be better than that of sigmoid in the deeper network; 
therefore, the gradient dispersion problem of sigmoid in the deeper network is solved successfully. (2) During training, Dropout is used to randomly ignore some neurons to avoid over-fitting of the model. (3) The overlapping maximum pooling is considered in CNN to avoid the blurring effect of average pooling. (4) The Local Response Normalization (LRN) layer is proposed to enhance the generalization ability of the model. (5) CUDA is used to accelerate the training of the CNN.
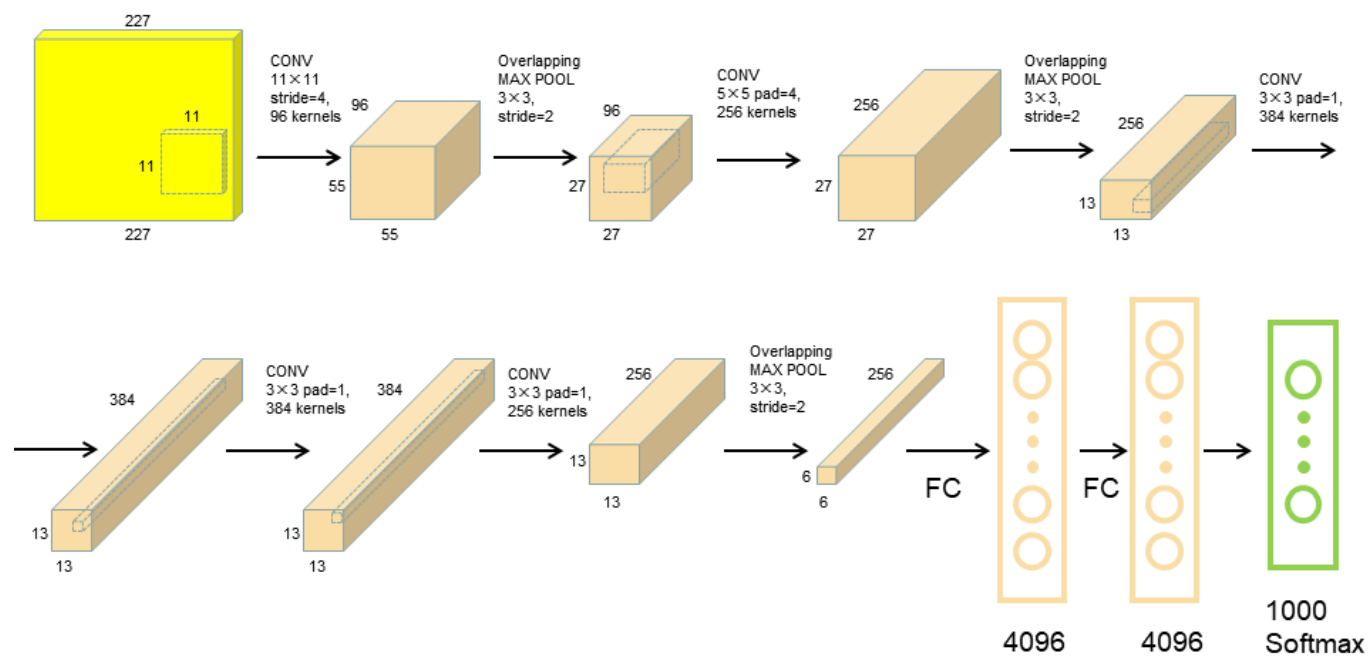

Figure 4. The network architecture of AlexNet [39].

Figure 5 provides the network architecture of VGG-16 [40]. VGG can be seen as a deeper version of AlexNet. The whole network is made up of a convolution layer and a full connection layer. Unlike AlexNet, VGG uses small convolution cores $(3 \times 3)$.

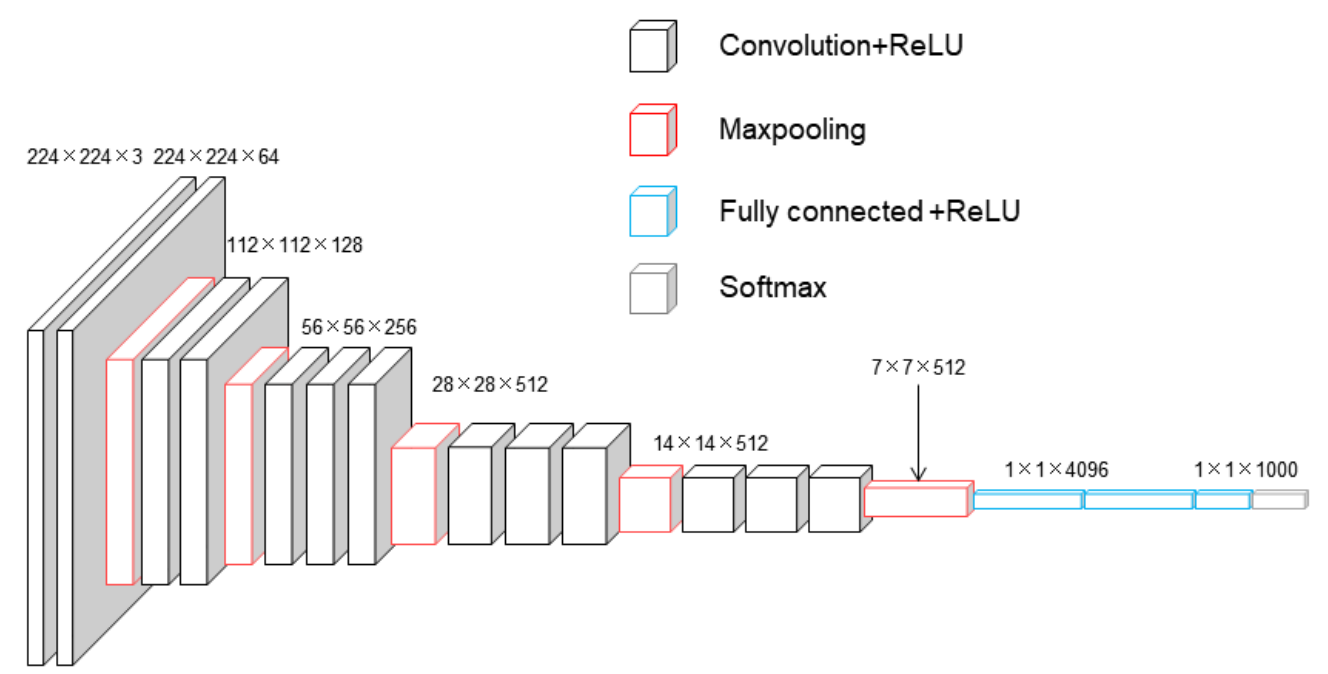

Figure 5. The network architecture of VGG-16 [40].

\subsection{The Training of the $\mathrm{CNNS}$}

The output layer of the LeNet network in this paper is activated by the sigmoid function. The Adam optimizer is used because the learning rate can be adjusted adaptively. The loss function uses a bivariate cross-entropy function. At the same time, to adapt to the shallow CNN and maintain the stable running of the model, the image size is reset to $36 \times 36$ when reading the picture (two-dimensional data). Pictures are processed in batches, and the number of pictures in a batch is 100 . 
Batch normalization (BN) is used for the AlexNet network instead of LRN. Unlike the LeNet network, the random gradient descent optimizer (SGD) is selected for the AlexNet network. Like the LeNet network, the cross-entropy function is considered the loss function of the AlexNet network and the probability of random deactivation is 0.5 . The algorithm resets the image size to $100 \times 100$ when extracting the picture, and cuts it to $99 \times 99$ in the center. The number of pictures is 100 in a batch.

For VGG-16, the BN and Adam optimizer are used. The loss function is still the binary cross-entropy function, and the random deactivation is used in the full connection layer with the probability 0.2 . The image size is reset to $100 \times 100$ when extracting the picture. The input data are processed in batches, but when affected by GPU memory, the number of batches is reduced to 50 .

\section{Simulations and Discussion}

In this section, the test results of the proposed CNN-based CSS scheme are presented and discussed. First of all, the detection probability and false alarm probability of the proposed schemes under the test set are given, where the SNR varies from $-8 \mathrm{~dB}$ to $5 \mathrm{~dB}$ with an SNR step of $1 \mathrm{~dB}$. Then, the sensing performance of the proposed scheme is compared with the AND rule, the OR rule and the MV rule.

Figures 6 and 7, respectively, provide the detection probability comparisons and the false alarm probability comparisons among the proposed CNN-based CSS schemes. From Figure 6, the detection probabilities of the three CNN-based CSS schemes are all above 0.9 , which indicates a stronger ability to protect the PU for the proposed schemes. In addition, the detection probability improves from LeNet to VGG-16. Based on the analysis in Section 4.2, the detection probability of the proposed CNN-based CSS schemes is gradually improved with the rise in the network depth. This indicates that the network depth helps with the feature acquisition of the primary signal and the sensing performance is at a high level in return. In Figure 7, the false alarm probability of the proposed CNNbased CSS schemes is always below 0.1, even below 0.05 for AlexNet and VGG-16, which indicates that the proposed schemes can keep the throughput of the cognitive system at a high level. In addition, the false alarm probability decreases gradually from LeNet to VGG-16. The same conclusion can be obtained from the detection probability-that the network depth contributes to decreasing the false alarm probability.

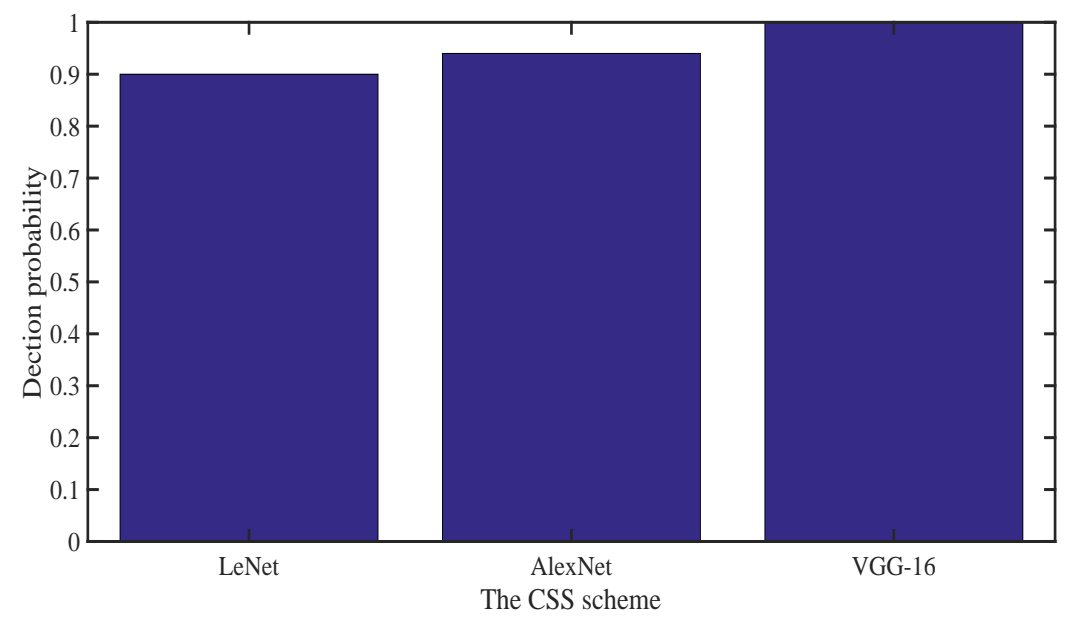

Figure 6. The detection probability comparisons of the CNN-based CSS schemes. 


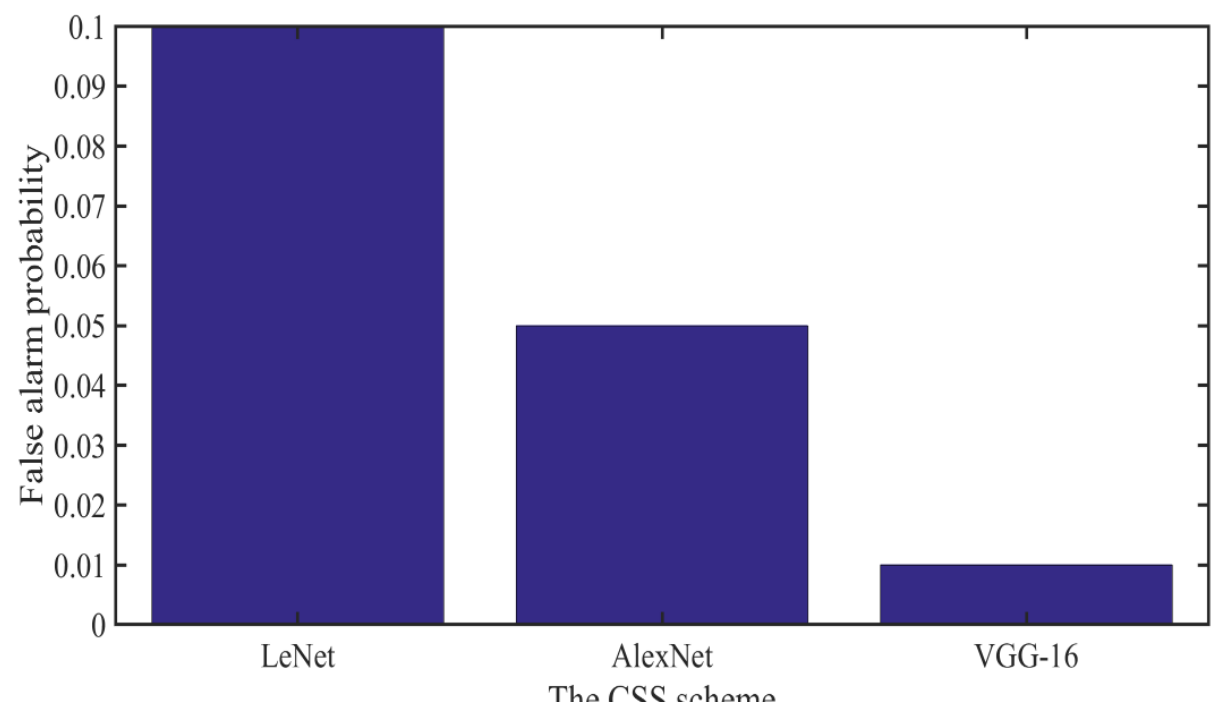

Figure 7. The false alarm probability comparisons of the CNN-based CSS schemes.

In Figure 8, the sensing performance comparisons are made among various CSS schemes, including the AND-, OR- and MV-based CSS scheme along with the proposed $\mathrm{CNN}$-based schemes. Five sensing nodes are considered in the experiments for convenience. From Figure 8, the detection probability of the MV-based CSS scheme is higher than that of the AND rule-based CSS scheme and the OR rule-based CSS scheme under the same false alarm probability, which validates the rationality of the simulation experiments. Moreover, the sensing performance of the proposed CNN-based CSS schemes is obviously higher than that of the classical schemes, which indicates that the proposed schemes are more effective for CSS. In addition, the detection probability of the VGG-16 network-based CSS scheme is highest, while the detection probability of the LeNet network is lowest under the same false alarm probability. This states that the network depth is positively related to the sensing performance of the proposed CNN-based CSS schemes, which corresponds with the analysis in Figures 6 and 7.

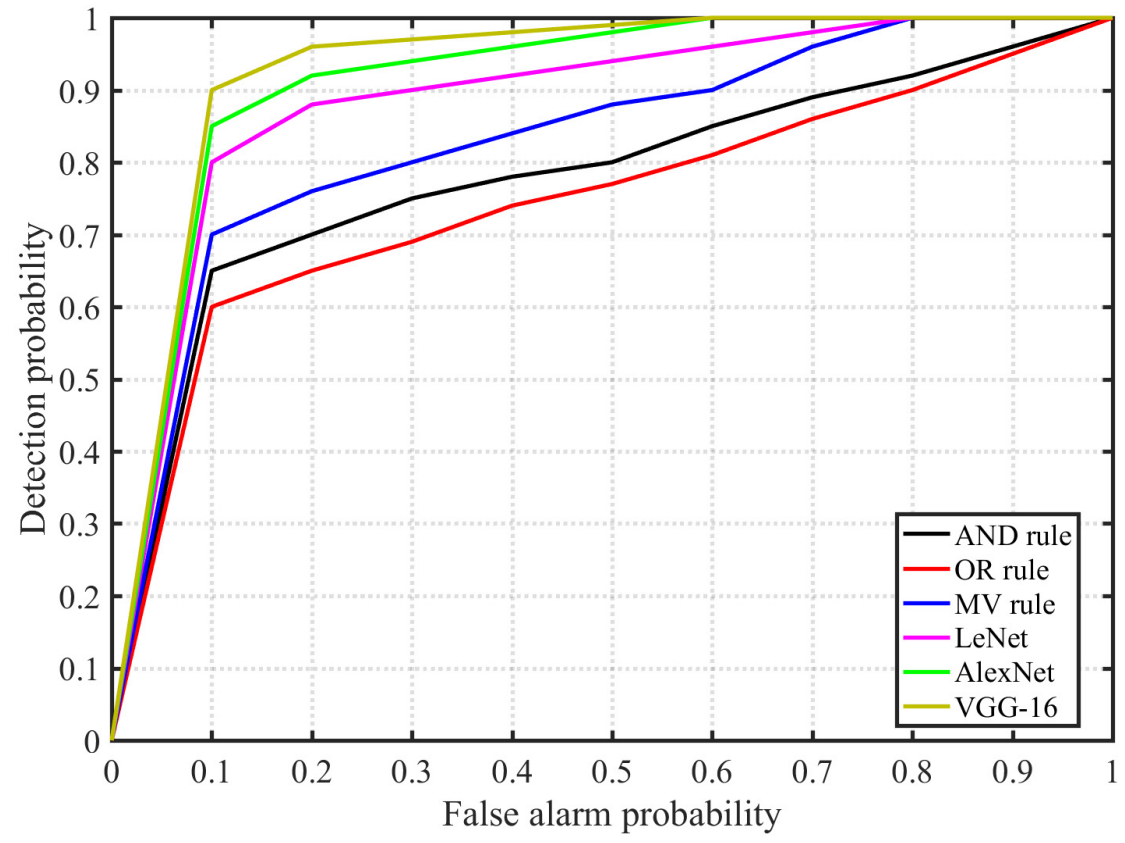

Figure 8. The sensing performance comparisons among various CSS schemes ( $\mathrm{SNR}=-5 \mathrm{~dB}$ ). 
As a supplement, the influence of the sensing node on the sensing performance is exhibited in Figure 9 based on the proposed AlexNet network-based CSS scheme, where the sensing node varies from 5 to 8 and from 8 to 10. From Figure 9, the sensing performance of the AlexNet network-based CSS scheme increases with the rise in the sensing node, which indicates that the sensing performance of the proposed CSS scheme is positively correlated with the sensing node.

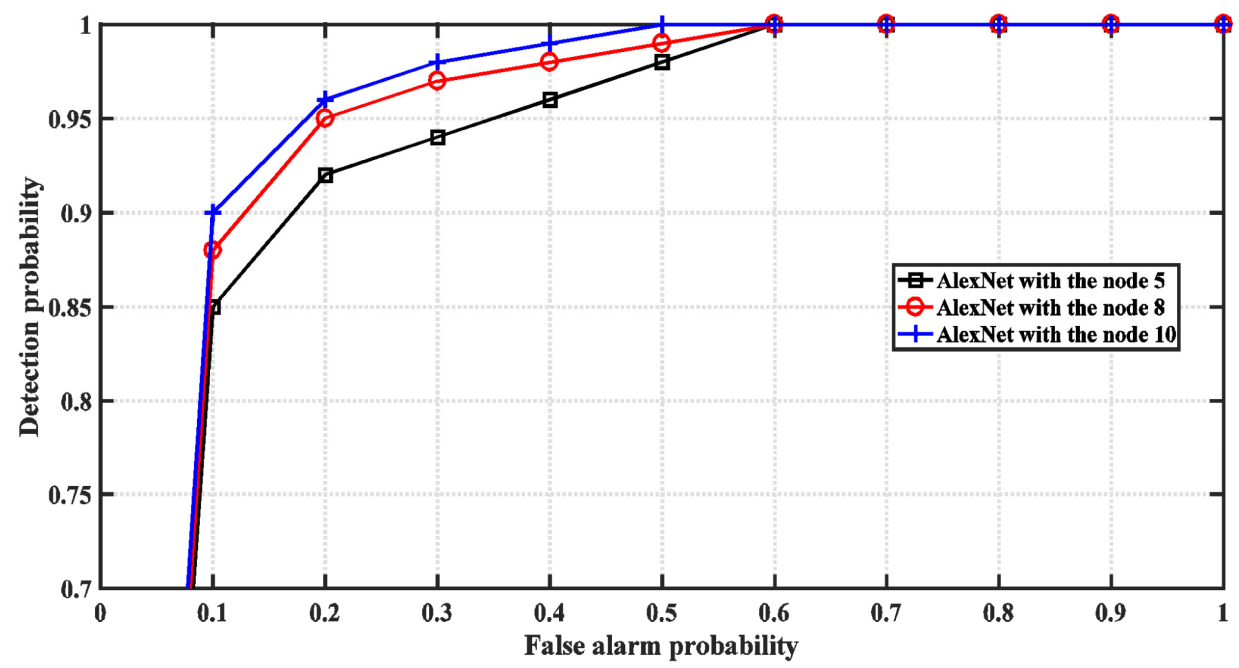

Figure 9. The influence of sensing node on the sensing performance.

Note that the receiver operator characteristic curve (ROC) is widely considered to exhibit the performance of SS, where the $X$ axis denotes the false alarm probability and the $\mathrm{Y}$ axis denotes the detection probability. In the simulations, the false alarm is fixed as $0-1$ with a step of 0.1 , where the corresponding detection probability is obtained by the mathematical statistics. The slope changes greatly at a false alarm rate of 0.1 because the false alarm probability jumps with the step of 0.1 . Specifically, the false alarm probability is 0 at first, while it will jump to 0.1 at the next moment for all the considered CSS schemes. As a result, all these plots show a similar pattern.

To further evaluate the performance of the proposed CNN-based CSS schemes, the average computation time is analyzed in Figure 10. Note that the computation time denotes the processing time of a picture through the CNN network, where the CNN network is trained in advance. In the simulations, many tests are conducted and the average computation time denotes the average value of the processing time for a picture. From Figure 9, the average computation time of the LeNet network-based CSS scheme is the lowest, while the average computation time of the VGG-16 network-based CSS scheme is the highest. This means that the sensing accuracy of the VGG-16 network-based CSS scheme is at the expense of the system overhead. As a result, the selection of the appropriate CNN-based CSS scheme depends on the system requirement of the sensing accuracy and the sensing speed. 


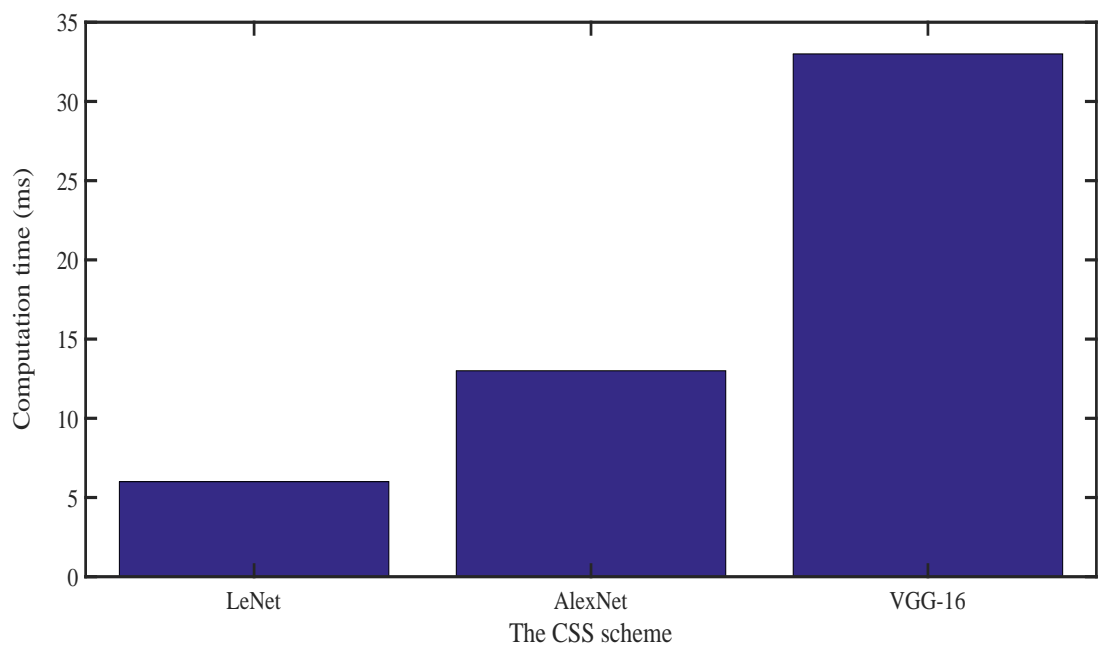

Figure 10. The average computation time of the proposed CSS schemes.

\section{Conclusions}

In this paper, three CNN-based CSS schemes are proposed to further improve the sensing performance of CSS. Firstly, the two-dimensional dataset is established based on the covariance matrix of the observed signal. Then, the LeNet, AlexNet and VGG-16-based CSS schemes are trained. Finally, the sensing performance of the proposed schemes is compared with that of the classical CSS schemes, including the AND, OR, majority voting rule. In addition, the average computation times of the proposed CNN-based CSS schemes are discussed. The simulation results show that the sensing performance of the proposed schemes is obviously higher than that of the classical schemes.

Author Contributions: Conceptualization, Y.T. and X.J.; methodology, Y.T.; validation, Y.T.; formal analysis, Y.T.; investigation, Y.T.; data curation, Y.T.; writing-original draft preparation, Y.T.; writing-review and editing, X.J. All authors have read and agreed to the published version of the manuscript.

Funding: This research received no external funding.

Institutional Review Board Statement: Not applicable.

Informed Consent Statement: Not applicable.

Data Availability Statement: The data presented in this study are available on request from the corresponding author. The data are not publicly available due to the commercial purpose.

Conflicts of Interest: The authors declare no conflict of interest.

\section{References}

1. Federal Communications Commission. Spectrum Policy Task Force Report; FCC Document ET Docket No. 02-155; Federal Communications Commission: Washington, DC, USA, November 2002.

2. Rawat, P.; Singh, K.D.; Bonnin, J.M. Cognitive radio for M2M and Internet of Things: A survey. Comput. Commun. 2016, 94, 1-29. [CrossRef]

3. Wu, Y.; Rong, B.; Salehian, K.; Gagnon, G. Cloud Transmission: A New Spectrum-Reuse Friendly Digital Terrestrial Broadcasting Transmission System. IEEE Trans. Broadcasting 2012, 58, 329-337. [CrossRef]

4. Wang, B.; Liu, K.R. Advances in cognitive radio networks: A survey. IEEE J. Sel. Top. Signal Process. 2011, 5, 5-23. [CrossRef]

5. Ku, M.-L.; Li, W.; Chen, Y.; Liu, K.J.R. Advances in Energy Harvesting Communications: Past, Present, and Future Challenges. IEEE Commun. Surv. Tutor. 2016, 18, 1384-1412. [CrossRef]

6. Ali, A.; Hamouda, W. Advances on Spectrum Sensing for Cognitive Radio Networks: Theory and Applications. IEEE Commun. Surv. Tutor. 2016, 19, 1277-1304. [CrossRef]

7. Axell, E.; Leus, G.; Larsson, E.G.; Poor, H.V. Spectrum Sensing for Cognitive Radio: State-of-the-Art and Recent Advances. IEEE Signal Process. Mag. 2012, 29, 101-116. [CrossRef] 
8. Zhang, H.; Jiang, C.; Beaulieu, N.C.; Chu, X.; Wang, X.; Quek, T.Q.S. Resource Allocation for Cognitive Small Cell Networks: A Cooperative Bargaining Game Theoretic Approach. IEEE Trans. Wirel. Commun. 2015, 14, 3481-3493. [CrossRef]

9. Ghasemi, A.; Sousa, E. Collaborative spectrum sensing for opportunistic access in fading environments. In Proceedings of the First IEEE International Symposium on New Frontiers in Dynamic Spectrum Access Networks, DySPAN 2005, Baltimore, MD, USA, 8-11 November 2005; pp. 131-136.

10. Salahdine, F.; El Ghazi, H.; Kaabouch, N.; Fihri, W.F. Matched filter detection with dynamic threshold for cognitive radio networks. In Proceedings of the 2015 International Conference on Wireless Networks and Mobile Communications (WINCOM), Marrakech, Morocco, 20-23 October 2015; pp. 1-6.

11. Ranjan, A.; Anurag; Singh, B. Design and analysis of spectrum sensing in cognitive radio based on energy detection. In Proceedings of the 2016 International Conference on Signal and Information Processing (IConSIP), Nanded, India, 6-8 October 2016; pp. 1-5.

12. Ilyas, I.; Paul, S.; Rahman, A.; Kundu, R.K. Comparative evaluation of cyclostationary detection based cognitive spectrum sensing. In Proceedings of the 2016 IEEE 7th Annual Ubiquitous Computing, Electronics \& Mobile Communication Conference (UEMCON), New York, NY, USA, 20-22 October 2016; pp. 1-7.

13. Zeng, Y.; Liang, Y.-C. Spectrum-Sensing Algorithms for Cognitive Radio Based on Statistical Covariances. IEEE Trans. Veh. Technol. 2009, 58, 1804-1815. [CrossRef]

14. Akyildiz, I.F.; Lo, B.F.; Balakrishnan, R. Cooperative spectrum sensing in cognitive radio networks: A survey. Phys. Commun. 2011, 4, 40-62. [CrossRef]

15. Na, W.; Yoon, J.; Cho, S.; Griffith, D.W.; Golmie, N. Centralized Cooperative Directional Spectrum Sensing for Cognitive Radio Networks. IEEE Trans. Mob. Comput. 2017, 17, 1260-1274. [CrossRef]

16. Chen, Z.; Gao, F. Cooperative-Generalized-Sensing-Based Spectrum Sharing Approach for Centralized Cognitive Radio Networks. IEEE Trans. Veh. Technol. 2015, 65, 3760-3764. [CrossRef]

17. Ling, Q.; Wen, Z.; Yin, W. Decentralized Jointly Sparse Optimization by Reweighted lq Minimization. IEEE Trans. Signal Process. 2013, 61, 1165-1170. [CrossRef]

18. Tsinos, C.G.; Berberidis, K. Decentralized Adaptive Eigenvalue-Based Spectrum Sensing for Multiantenna Cognitive Radio Systems. IEEE Trans. Wirel. Commun. 2014, 14, 1703-1715. [CrossRef]

19. Chaudhari, S.; Koivunen, V.; Poor, H.V. Autocorrelation-Based Decentralized Sequential Detection of OFDM Signals in Cognitive Radios. IEEE Trans. Signal Process. 2009, 57, 2690-2700. [CrossRef]

20. Di Lorenzo, P.; Barbarossa, S.; Sayed, A.H. Bio-Inspired Decentralized Radio Access Based on Swarming Mechanisms Over Adaptive Networks. IEEE Trans. Signal Process. 2013, 61, 3183-3197. [CrossRef]

21. Noorshams, N.; Malboubi, M.; Bahai, A. Centralized and decentralized cooperative spectrum sensing in cognitive radio networks: A novel approach. In Proceeding of the 2010 IEEE 11th International Workshop on Signal Processing Advances in Wireless Communications (SPAWC), Marrakech, Morocco, 20-23 June 2010; pp. 1-5. [CrossRef]

22. Shinde, S.C.; Jadhav, A.N. Centralized cooperative spectrum sensing with energy detecion in cognitive radio and optimization. In Proceedings of the 2016 IEEE International Conference on Recent Trends in Electronics, Information \& Communication Technology (RTEICT), Bangalore, India, 20-21 May 2016; pp. 1002-1006.

23. Misra, R.; Kannu, A.P. Optimal sensing-order in cognitive radio networks with cooperative centralized sensing. In Proceedings of the 2012 IEEE International Conference on Communications (ICC), Ottawa, ON, Canada, 10-15 June 2012; pp. 1566-1570.

24. Bastami, B.A.; Saberinia, E. A low complexity multi-threshold centralized detection strategy for cooperative spectrum sensing. In Proceedings of the 2011 IEEE 22nd International Symposium on Personal, Indoor and Mobile Radio Communications, Toronto, ON, Canada, 11-14 September 2011; pp. 349-353.

25. Gao, J.; Yi, X.; Zhong, C.; Chen, X.; Zhang, Z. Deep Learning for Spectrum Sensing. IEEE Wirel. Commun. Lett. 2019, 8, 1727-1730. [CrossRef]

26. He, H.; Jiang, H. Deep Learning Based Energy Efficiency Optimization for Distributed Cooperative Spectrum Sensing. IEEE Wirel. Commun. 2019, 26, 32-39. [CrossRef]

27. Liu, H.; Zhu, X.; Fujii, T. Ensemble Deep Learning Based Cooperative Spectrum Sensing with Stacking Fusion Center. In Proceedings of the 2018 Asia-Pacific Signal and Information Processing Association Annual Summit and Conference (APSIPA ASC), Honolulu, HI, USA, 12-15 November 2018; pp. 1841-1846.

28. Zeng, Y.; Zhang, M.; Han, F.; Gong, Y.; Zhang, J. Spectrum Analysis and Convolutional Neural Network for Automatic Modulation Recognition. IEEE Wirel. Commun. Lett. 2019, 8, 929-932. [CrossRef]

29. Liu, F.; Zhou, Y.; Liu, Y. A Deep Neural Network Method for Automatic Modulation Recognition in OFDM with Index Modulation. In Proceedings of the 2019 IEEE 89th Vehicular Technology Conference (VTC2019-Spring), Kuala Lumpur, Malaysia, 28 April-1 May 2019; pp. 1-5.

30. Chen, Z.; Xu, Y.-Q.; Wang, H.; Guo, D. Deep STFT-CNN for Spectrum Sensing in Cognitive Radio. IEEE Commun. Lett. 2021, 25, 864-868. [CrossRef]

31. Tavares, C.H.A.; Filho, J.C.M.; Proença, M.L., Jr.; Abrão, T. Machine learn-ing-based models for spectrum sensing in cooperative radio networks. IET Commun. 2020, 14, 3102-3109. [CrossRef]

32. Xie, J.; Fang, J.; Liu, C.; Li, X. Deep Learning-Based Spectrum Sensing in Cognitive Radio: A CNN-LSTM Approach. IEEE Commun. Lett. 2020, 24, 2196-2200. [CrossRef] 
33. Cai, P.; Zhang, Y.; Pan, C. Coordination Graph-Based Deep Reinforcement Learning for Cooperative Spectrum Sensing Under Correlated Fading. IEEE Wirel. Commun. Lett. 2020, 9, 1778-1781. [CrossRef]

34. Shi, Z.; Gao, W.; Zhang, S.; Liu, J.; Kato, N. Machine Learning-Enabled Cooperative Spectrum Sensing for Non-Orthogonal Multiple Access. IEEE Trans. Wirel. Commun. 2020, 19, 5692-5702. [CrossRef]

35. Sarikhani, R.; Keynia, F. Cooperative Spectrum Sensing Meets Machine Learning: Deep Reinforcement Learning Ap-proach. IEEE Commun. Lett. 2020, 24, 1459-1462. [CrossRef]

36. Lim, C.H.; Guimaraes, D.A. GLRT-Based Spectrum Sensing Techniques for Pulse Radar Signals. IEEE Commun. Lett. 2019, 24, 447-450. [CrossRef]

37. Meng, X.; Inaltekin, H.; Krongold, B. End-to-End Deep Learning-Based Compressive Spectrum Sensing in Cognitive Radio Networks. In Proceedings of the ICC 2020-2020 IEEE International Conference on Communications (ICC), Dublin, Ireland, 7-11 June 2020; pp. 1-6.

38. Bottou, L.; Cortes, C.; Denker, J.; Drucker, H.; Guyon, I.; Jackel, L.; LeCun, Y.; Muller, U.; Sackinger, E.; Simard, P.; et al. Comparison of classifier methods: A case study in handwritten digit recognition. In Proceedings of the IEEE 12th IAPR International Conference on Pattern Recognition (Cat. No.94CH3440-5), Jerusalem, Israel, 9-13 October 2002; Volume 3, pp. 77-82.

39. Lv, M.; Zhou, G.; He, M.; Chen, A.; Zhang, W.; Hu, Y. Maize Leaf Disease Identification Based on Feature Enhancement and DMS-Robust Alexnet. IEEE Access 2020, 8, 57952-57966. [CrossRef]

40. Alippi, C.; Disabato, S.; Roveri, M. Moving Convolutional Neural Networks to Embedded Systems: The AlexNet and VGG-16 Case. In Proceedings of the 2018 17th ACM/IEEE International Conference on Information Processing in Sensor Networks (IPSN), Porto, Portugal, 11-13 April 2018; pp. 212-223. 